\title{
Magnetic Metamaterials at Telecommunication and Visible Frequencies
}

\author{
C. Enkrich, ${ }^{1}$ M. Wegener, ${ }^{1,2}$ S. Linden, ${ }^{2}$ S. Burger, ${ }^{3}$ L. Zschiedrich, ${ }^{3}$ F. Schmidt,${ }^{3}$ J. F. Zhou, ${ }^{4}$ \\ Th. Koschny, ${ }^{4}$ and C. M. Soukoulis ${ }^{4,5}$ \\ ${ }^{1}$ Institut für Angewandte Physik, Universität Karlsruhe (TH), D-76131 Karlsruhe, Germany \\ ${ }^{2}$ Institut für Nanotechnologie, Forschungszentrum Karlsruhe in der Helmholtz-Gemeinschaft, D-76021 Karlsruhe, Germany \\ ${ }^{3}$ Zuse Institute Berlin, Takustraße 7, D-14195 Berlin, Germany, \\ and DFG Forschungszentrum MATHEON, Straße des 17. Juni 136, 10623 Berlin, Germany \\ ${ }^{4}$ Ames Laboratory and Department of Physics and Astronomy, Iowa State University, Ames, Iowa 50011, USA \\ ${ }^{5}$ Institute of Electronic Structure and Laser, FORTH and Department of Materials Science and Technology, \\ 71110 Heraklion, Crete, Greece
}

(Received 28 April 2005; published 7 November 2005)

\begin{abstract}
Arrays of gold split rings with a 50-nm minimum feature size and with an $L C$ resonance at $200 \mathrm{THz}$ frequency (1.5 $\mu \mathrm{m}$ wavelength) are fabricated. For normal-incidence conditions, they exhibit a pronounced fundamental magnetic mode, arising from a coupling via the electric component of the incident light. For oblique incidence, a coupling via the magnetic component is demonstrated as well. Moreover, we identify a novel higher-order magnetic resonance at around $370 \mathrm{THz}(800 \mathrm{~nm}$ wavelength) that evolves out of the Mie resonance for oblique incidence. Comparison with theory delivers good agreement and also shows that the structures allow for a negative magnetic permeability.
\end{abstract}

DOI: 10.1103/PhysRevLett.95.203901

In usual crystals, the atoms are arranged in a periodic fashion with lattice constants less than $1 \mathrm{~nm}$. This is orders of magnitude smaller than the wavelength of visible light. Thus, the light experiences an effective homogeneous material; it does not "see" the underlying periodicity (apart from crystal symmetries). Microscopically, the light excites electric dipoles that reradiate with a certain retardation, slowing down the phase velocity of light in the material by a factor called the index of refraction. Metamaterials are artificial periodic structures with "lattice constants" that are still smaller than the wavelength of light. Again, the light field "sees" an effective homogeneous material. The "atoms," however, are not real atoms but are rather artificial nanostructures composed of many atoms. This allows for tailoring their properties in a way not possible with normal atoms. Indeed, Pendry [1] showed that a combination of "magnetic atoms" and "electric atoms" (i.e., split rings and metallic wires) with negative permeability $\mu$ and permittivity $\epsilon$, respectively, can lead to materials with a negative index of refraction $n$ [2]. These materials open a whole new chapter of photonics connected with novel concepts and potential applications $[3,4]$.

The main technological challenge is to obtain a negative permeability $\mu<0$ at telecom or visible frequencies, which does not occur in natural materials. Starting with first demonstrations in the microwave regime [5], the achieved magnetic resonance frequencies have increased by more than 4 orders of magnitude over the last four years [5-10], reaching a record of $100 \mathrm{THz}(3 \mu \mathrm{m}$ wavelength) in November 2004 [7].

So far, the magnetic atom of choice has been the split ring resonator (SRR), in essence just a small $L C$ circuit consisting of an inductance $L$ and a capacitance $C$. The resonance frequency of this $L C$ circuit scales inversely
PACS numbers: 42.70.-a, 42.25.-p, 78.20.Ci

with its size, provided the frequencies are significantly below the metal plasma frequency. Near the resonance, the current in the inductance can lead to a magnetic field opposing the external magnetic field of the light, hence enabling $\mu<0$.

The design used here closely follows our recent approach based on arrays of single SRR [7,11]. The structures are fabricated using standard electron-beam lithography on a $1 \mathrm{~mm}$ thick glass substrate coated with a $5 \mathrm{~nm}$ thin film of indium-tin-oxide (ITO), in order to avoid charging effects of the poly(methyl methacrylate) resist layer (PMMA $950 \mathrm{k}$ ) during the exposure. The gold film thickness is $30 \mathrm{~nm}$. To increase the resonance frequency at a given minimum feature size and to simplify the nanofabrication, we almost eliminate the tiny upper arms of the SRR, leading to more " $U$ "-shaped structures [see Fig. 1(a)]. Intuitively, these $U$ s correspond to $\frac{3}{4}$ of one winding of a magnetic coil with inductance $L$. The ends of the $U$-shaped wire form the capacitance $C$. We employ $(100 \mu \mathrm{m})^{2}$ periodic quadratic arrays of such SRR with the dimensions apparent from the electron micrographs in Fig. 1.

Figure 1 connects to our previous work [7] and shows spectra taken under normal incidence with a commercial Fourier-transform microscope spectrometer [12]. If the incident light is polarized horizontally, the electric field can couple to the capacitance of the SRR and induce a circulating current in the coil leading to a magnetic-dipole moment normal to the SRR plane. Note that this resonance at $1.5 \mu \mathrm{m}$ wavelength is yet more pronounced than in our previous work [7] at $3 \mu \mathrm{m}$, due mainly to the increased ratio between thickness and lateral size of the SRR. The magnetic resonance disappears for vertical incident polarization [Fig. 1(c)], leaving behind only the Mie resonance 
(a)

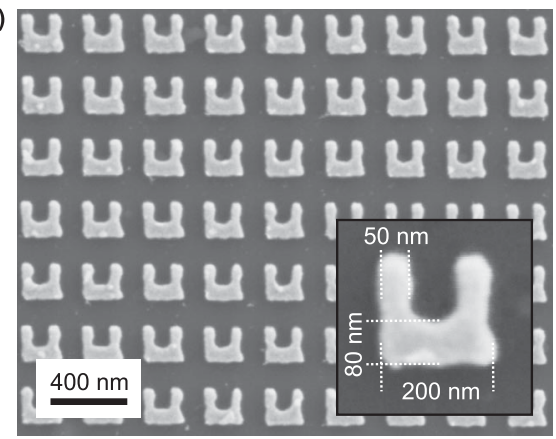

(b)

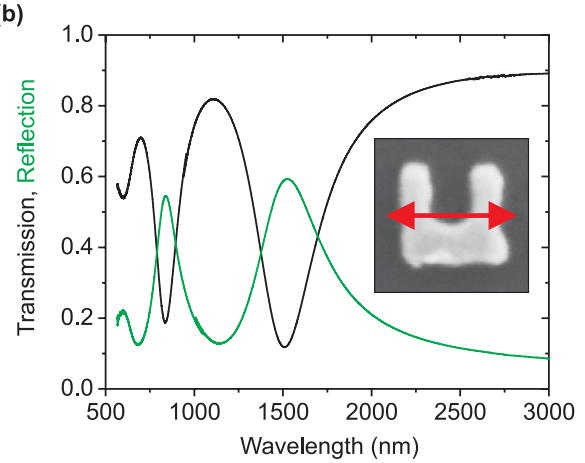

(c)

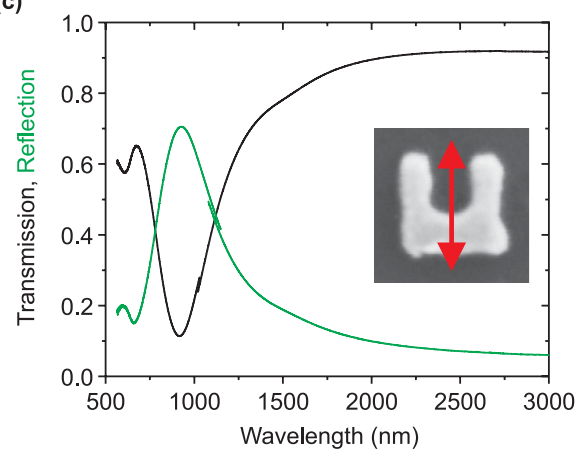

FIG. 1 (color). Electron micrograph of a split ring array with a total size of $(100 \mu \mathrm{m})^{2}$. The lower right-hand side inset shows the dimensions of an individual split ring. The corresponding measured normal-incidence transmission and reflection spectra for horizontal and vertical polarization are shown in (b) and (c), respectively. For (b), one can couple to the fundamental magnetic mode at $1.5 \mu \mathrm{m}$ wavelength via the electric-field component of the incident light; for (c), one cannot.

of the SRR around $950 \mathrm{~nm}$ wavelength. As this resonance will become important below as well, we briefly recapitulate its physics. Here the electric field of the light leads to a charge accumulation at the surfaces of the vertical SRR arms, resulting in a depolarization field. Depending on the permittivity of the metal, hence depending on the frequency of light, this depolarization field can enhance or suppress the external electric field. (We also observe a weaker short-wavelength Mie resonance around $600 \mathrm{~nm}$ in Fig. 1, which is due to the depolarization field of the short axis, i.e., the width of the SRR arms.) Notably, the fundamental Mie resonance of our SRR changes in spectral position and width between the two different polarization configurations. This can be understood as follows: For horizontal incident polarization and for the frequencies of interest here, only the fundamental Mie resonance of the SRR bottom arm is excited. For vertical polarization, the two similarly shaped vertical SRR arms contribute. The latter are coupled via the SRR's bottom arm (and via the radiation field). As usual, the coupling of two degenerate modes leads to an avoided crossing with two new effective oscillation modes, a symmetric and an antisymmetric one, which are frequency down-shifted and up-shifted as compared to the uncoupled resonances, respectively. The antisymmetric mode cannot be excited at all for normal incidence as it has zero effective electric-dipole moment. The redshifted symmetric mode can be excited. It even has a larger effective electric-dipole moment than a single arm.

The optical response of SRR is not only polarizationdependent but also highly anisotropic. Thus, we have performed transmission experiments under oblique incidence (Fig. 2) using a dedicated homebuilt setup [12]. Compared with the Fourier-transform microscope spectrometer used above, this setup has improved polarization optics by using Glan-Thomson polarizers and a smaller
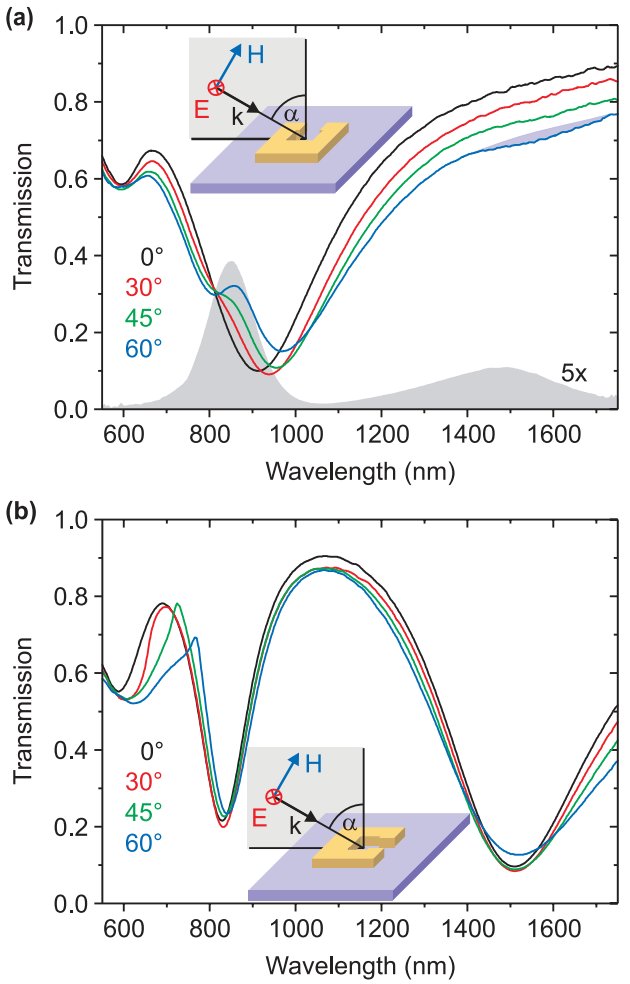

FIG. 2 (color). Measured transmission spectra taken for oblique incidence for the configurations shown as insets (where $\alpha=60^{\circ}$ ). In (a), coupling to the fundamental magnetic mode at $1.5 \mu \mathrm{m}$ wavelength is only possible via the magnetic-field component of the incident light; for (b), both electric and magnetic field can couple. Note the small but significant feature in (a) for $60^{\circ}$ around $1.5 \mu \mathrm{m}$ wavelength. The lower gray area in (a) is the transmission into the linear polarization orthogonal to the incident one for $\alpha=60^{\circ}$. We argue that this observable can be viewed as a fingerprint of magnetic resonances under these conditions. 
effective opening angle ( \pm 7 degrees). It still allows one to investigate a sample of size $(100 \mu \mathrm{m})^{2}$ with a large angle of incidence with respect to the surface normal over a broad spectral range. In Fig. 2(a), the electric component of the incident light cannot couple to the $L C$ circuit resonance for any angle. With increasing angle, however, the magnetic field acquires a component normal to the SRR plane. This component can induce a circulating electric current in the SRR coil via the induction law [see righthand side inset in Fig. 3(a)]. This current again leads to a magnetic-dipole moment normal to the SRR plane, which can counteract the external magnetic field. The magnitude of this resonance (highlighted by the blue area around $1.5 \mu \mathrm{m}$ wavelength) is indeed consistent with theory (see below) and leads to an effective negative magnetic permeability for propagation in the SRR plane and for a stack of SRR layers rather than just one layer considered here. This aspect has been verified explicitly by retrieving the effective permittivity and permeability from the calculated transmission and reflection spectra [13,14]. The shape of the retrieved magnetic permeability closely resembles that published in our previous work [7] at $3 \mu \mathrm{m}$ wavelength. It exhibits a negative permeability with a minimum value of $\mu=-0.25$ at $1.67 \mu \mathrm{m}$ wavelength [12]. This value could be further improved by increasing the number of SRR per
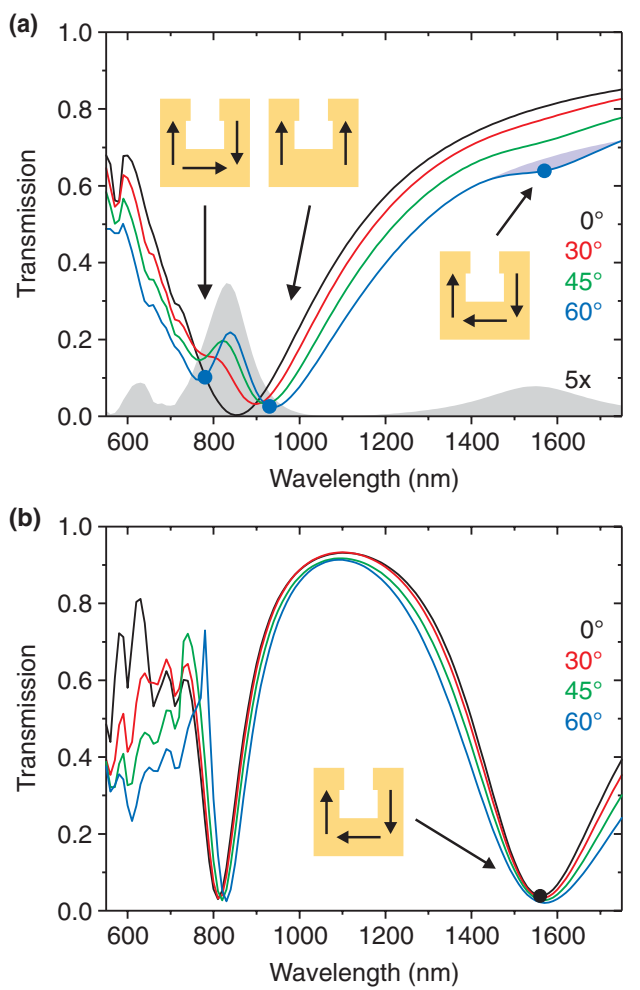

FIG. 3 (color). Calculated transmission spectra for different angles on the same scale as the experiment (Fig. 2). The polarization geometries in (a) and (b) are identical to those in Figs. 2(a) and 2(b), respectively. The insets schematically show the current distributions at the frequencies and angles marked by the dots. area [compare Fig. 1(a)], hence increasing the "oscillator strength" of the magnetic resonance.

Another striking feature of the spectra in Fig. 2(a) is that the $950 \mathrm{~nm}$ wavelength Mie resonance at normal incidence splits into two resonances for oblique incidence. This aspect can be understood continuing along the lines of our above intuitive discussion: For finite angles of incidence, the phase fronts of the electric field are tilted with respect to the SRR plane. Thus, the vertical SRR arms are excited with a small but finite time delay, equivalent to a finite phase shift. This shift allows coupling to the antisymmetric mode of the coupled system of the two vertical arms as well. In one half-cycle of light, one gets a positive charge at the lower left-hand side corner of the SRR and a negative charge at the lower right-hand side corner, resulting in a compensating current in the horizontal bottom arm. Characteristic snapshots of the current distributions in the SRR are schematically shown as insets in Fig. 3(a). Altogether, we get a part of a circulating current, leading to a magnetic-dipole moment. This type of magnetic resonance has not been observed before. Its spectral position of $800 \mathrm{~nm}$ at $60^{\circ}$ angle in Fig. 2(a) is just within the visible regime - for the first time, one can literally see a magnetic resonance.

According to our reasoning for oblique incidence (e.g., $60^{\circ}$ ), we expect a circulating current component for wavelengths near the two magnetic resonances at $1.5 \mu \mathrm{m}$ and $800 \mathrm{~nm}$, respectively. Any circulating current is evidently connected with a current in the horizontal bottom arm of the SRR. According to the usual laws of a Hertz dipole, the corresponding charge oscillation in the bottom arm can radiate into the forward direction with an electric-field component orthogonal to the incident polarization. In other words, for oblique incidence, the fingerprint of the magnetic resonances is a rotation of polarization. Such rotation is indeed unambiguously observed in our experiments [see gray area in Fig. 2(a)], strongly supporting our above interpretation.

Figure 2(b) shows spectra for a second polarization configuration in which both the electric and the magnetic components of the light field can couple to the SRR for oblique incidence. The same configuration was used in Ref. [6]. Our results immediately show that coupling to the fundamental magnetic resonance at $1.5 \mu \mathrm{m}$ wavelength is not mediated by the magnetic field alone here. The two further independent polarization and angle configurations are consistent with our above interpretation but do not reveal any additional resonances [12].

In order to further strengthen our above interpretation of the different resonances, we compare the measured spectra with theory. It turns out that numerical calculation of spectra for oblique incidence is much more of a challenge than for normal incidence or for propagation in the plane of the SRR array. Thus, we have not only followed our previous finite-difference time-domain approach [7] but also an advanced frequency-domain based finite-element approach. We discretize the geometry of a unit cell 
$(315 \mathrm{~nm} \times 330 \mathrm{~nm} \times 60 \mathrm{~nm})$ including the SRR and the surrounding layered media with an unstructured mesh of tetrahedra. The coarse mesh is automatically refined to a fine mesh consisting about 6000 tetrahedra. Bloch-periodic boundary conditions are applied in the $x$ and $y$ directions [15] with lattice constants of $a_{x}=315 \mathrm{~nm}$ and $a_{y}=$ $330 \mathrm{~nm}$, respectively. In the $\pm z$ direction, we apply transparent boundary conditions [16]. To avoid dealing with the complex SRR shape apparent from Fig. 1, the three SRR arms are approximated as rectangles with a width of $65 \mathrm{~nm}$ for the two vertical arms and $90 \mathrm{~nm}$ for the bottom arm. These values have been fine-tuned to fit the calculated to the measured resonance positions. The SRR side length of $200 \mathrm{~nm}$ and the gold thickness of $30 \mathrm{~nm}$ are identical to the values already quoted above. For the permittivity of gold, we assume a wavelength dependence according to the Drude model, with a plasma frequency of $\omega_{\mathrm{pl}}=1.367 \times$ $10^{16} \mathrm{~s}^{-1}$ and a collision frequency of $\omega_{\mathrm{c}}=6.478 \times$ $10^{13} \mathrm{~s}^{-1}$. The permittivity of the ITO layer (glass substrate) is taken as 3.8 (2.25). We discretize Maxwell's equations using vectorial finite elements (Whitney elements) of second polynomial order. The resulting sparse matrix equation (with about 130000 unknowns corresponding to the solution on the fine mesh) is solved on a standard personal computer by either standard linear algebra decomposition techniques or multigrid algorithms, depending on the problem size.

Figure 3 exhibits calculated spectra for different angles of incidence. The graphical representation is identical to that of the experiment (see Fig. 2). Obviously, the qualitative agreement between experiment and theory is excellent. Especially the spectral positions as well as the magnitudes of the different resonances and the rotation of the polarization [gray area at the bottom of Fig. 3(a)] are well reproduced. For normal incidence, the spectra calculated with the finite-element frequency-domain approach agree well with those obtained from the finite-difference timedomain simulations, which are used for the retrieval procedure (see above). The schematic insets shown in Fig. 3 have been derived from the movies [12] of the calculated field distributions and correspond to the qualitative discussion given above.

In conclusion, normal optical materials exhibit only an electric-dipole response. Here we have demonstrated pronounced magnetic-dipole modes at $1.5 \mu \mathrm{m}$ and at $800 \mathrm{~nm}$ wavelength, respectively. The one at $1.5 \mu \mathrm{m}$ is the "usual" $L C$ resonance of the split ring resonators and would lead to a negative magnetic permeability indeed. The one at $800 \mathrm{~nm}$ wavelength is a higher-order magnetic resonance, which is identified here for the first time. On the one hand, the nanofabrication of magnetic metamaterials at these frequencies requires much more of a technological effort than at microwave frequencies. On the other hand, linear optical spectroscopy can be performed much more conveniently and in a more controlled fashion. Moreover, the availability of lasers in this spectral regime enables future nonlinear optical experiments.

We acknowledge the support by the DFG-Center for Functional Nanostructures within Subproject No. A 1.5, by DFG Project No. We-1497/9-1, by DFG priority programme SPP 1113, and by the BMBF within Project No. 13N8252. The research of C. M. S. is further supported by the Alexander von Humboldt Foundation, by Ames Laboratory (Contract No. W-7405-Eng-82), EU FET project DALHM and DARPA (Contract No. MDA 97201-2-0016).

Note added in proof.-Similar $U$-shaped SRR have recently also been discussed theoretically in Ref. [17].

[1] J. B. Pendry, A. J. Holden, D. J. Robbins, and W. J. Stewart, IEEE Trans. Microwave Theory Tech. 47, 2075 (1999).

[2] V. G. Veselago, Sov. Phys. Usp. 10, 509 (1968).

[3] J. B. Pendry, Phys. Rev. Lett. 85, 3966 (2000).

[4] D. R. Smith, J. B. Pendry, and M. C. K. Wiltshire, Science 305, 788 (2004).

[5] R. A. Shelby, D. R. Smith, and S. Schultz, Science 292, 77 (2001).

[6] T. J. Yen, W. J. Padilla, N. Fang, D. C. Vier, D. R. Smith, J. B. Pendry, D. N. Basov, and X. Zhang, Science 303, 1494 (2004).

[7] S. Linden, C. Enkrich, M. Wegener, J. Zhou, T. Koschny, and C. M. Soukoulis, Science 306, 1351 (2004).

[8] S. Zhang, W. Fan, B. K. Minhas, A. Frauenglass, K. J. Malloy, and S. R. J. Brueck, Phys. Rev. Lett. 94, 037402 (2005).

[9] H. O. Moser, B. D. F. Casse, O. Wilhelmi, and B. T. Saw, Phys. Rev. Lett. 94, 063901 (2005).

[10] N. Katsarakis, G. Konstantinidis, A. Kostopoulos, R. S. Penciu, T.F. Gundogdu, M. Kafesaki, E. N. Economou, Th. Koschny, and C. M. Soukoulis, Opt. Lett. 30, 1348 (2005).

[11] N. Katsarakis, Th. Koschny, M. Kafesaki, E. N. Economou, and C. M. Soukoulis, Appl. Phys. Lett. 84, 2943 (2004).

[12] See EPAPS document No. E-PRLTAO-95-075545 for additional information on the optical characterization of the SRR arrays and on the retrieval procedure. This document can be reached via a direct link in the online article's HTML reference section or via the EPAPS homepage (http://www.aip.org/pubservs/epaps.html).

[13] D. R. Smith, S. Schultz, P. Markos, and C. M. Soukoulis, Phys. Rev. B 65, 195104 (2002).

[14] Th. Koschny, P. Markos, E. N. Economou, D. R. Smith, D. C. Vier, and C. M. Soukoulis, Phys. Rev. B 71, 245105 (2005).

[15] S. Burger, R. Klose, A. Schädle, F. Schmidt, and L. Zschiedrich, Proc. SPIE-Int. Soc. Opt. Eng. 5728, 164 (2005).

[16] L. Zschiedrich, R. Klose, A. Schädle, and F. Schmidt, J. Comput. Appl. Math. (to be published).

[17] V. A. Podolskiy, A. K. Sarychev, E. E. Narimanov, and V. M. Shalaev, J. Opt. A Pure Appl. Opt. 7, S32 (2005). 